\title{
"Transport Me Away": Fostering Flow in Open Offices through Virtual Reality
}

\author{
Anastasia Ruvimova \\ University of Zurich, \\ Switzerland \\ anastasia.ruvimova@uzh.ch
}

\author{
Junhyeok Kim \\ University of Waterloo, \\ Canada \\ junhyeok.kim@uwaterloo.ca
}

\author{
Thomas Fritz \\ University of Zurich, \\ Switzerland \\ fritz@ifi.uzh.ch
}

\author{
Mark Hancock \\ University of Waterloo, \\ Canada \\ mark.hancock@uwaterloo.ca
}

\author{
David C. Shepherd \\ Virginia Commonwealth \\ University, USA \\ shepherdd@vcu.edu
}

\begin{abstract}
Open offices are cost-effective and continue to be popular. However, research shows that these environments, brimming with distractions and sensory overload, frequently hamper productivity. Our research investigates the use of virtual reality (VR) to mitigate distractions in an open office setting and improve one's ability to be in flow. In a lab study, 35 participants performed visual programming tasks in four combinations of physical (open or closed office) and virtual environments (beach or virtual office). While participants both preferred and were in flow more in a closed office without VR, in an open office, the VR environments outperformed the no VR condition in all measures of flow, performance, and preference. Especially considering the recent rapid advancements in VR, our findings illustrate the potential VR has to improve flow and satisfaction in open offices.
\end{abstract}

\section{Author Keywords}

Virtual reality; open offices; flow; work.

\section{CCS Concepts}

-Human-centered computing $\rightarrow$ Human computer interaction (HCI); Virtual reality; User studies;

\section{INTRODUCTION}

For knowledge workers, especially software developers, flow [13] — characterized by energized focus and complete engagement on a given task-usually leads to increased productivity and personal development, even improving life satisfaction. While achieving flow is desirable, it can be challenging, as it requires an environment, that is "free from distractions" [48] for long periods of time [18,41]. Unfortunately,

Permission to make digital or hard copies of all or part of this work for personal or classroom use is granted without fee provided that copies are not made or distributed for profit or commercial advantage and that copies bear this notice and the full citation on the first page. Copyrights for components of this work owned by others than the author(s) must be honored. Abstracting with credit is permitted. To copy otherwise, or republish, to post on servers or to redistribute to lists, requires prior specific permission and/or a fee. Request permissions from permissions@ acm.org. CHI '20, April 25-30, 2020, Honolulu, HI, USA.

(C) 2020 Copyright is held by the owner/author(s). Publication rights licensed to ACM. ACM ISBN 978-1-4503-6708-0/20/04 ...\$15.00.

DOI: https://dx.doi.org/10.1145/3313831.3376724 this particular condition is becoming harder and harder to realize, as modern office life is fraught with distractions. Text messages, colleagues' questions, meetings, nearby phone conversations, and myriad other stimuli bombard office workers with constant interruptions [2,38].

Because distractions are known to reduce productivity, it seems natural that employers would provide their knowledge workers with closed offices to minimize distractions. Why then are many employers moving to the open office concept, especially when it is known to cause distractions [7]? Unfortunately, because open offices are much cheaper than traditional, private offices, there is a clear incentive for employers to move to open offices. "Managers present [moving to open offices] as necessary for greater collaboration and productivity, but $99 \%$ of the changes are really driven by the desire to cut costs" [62].

Fortunately, at the same time that knowledge workers are being forced into distraction-filled open offices, virtual reality hardware is rapidly developing, presenting a potential solution. What if employers could continue to implement open office layouts, which save money, while, at the same time, provide a virtually closed office that offers employees many of the benefits of a traditional closed office?

Until recently, this concept was a cyberpunk [25] fantasy; headset resolution was low, causing text to be difficult to read [19], and headset tracking was poor, causing motion sickness in many participants [31]. However, recent advances have not only addressed these shortcomings, they have been dramatic enough to show that, within a few years, VR technology may improve drastically. For instance, the recently released HP Reverb offers the unprecedented resolution of $2160 \times 2160$ pixels per eye, making it possible to comfortably read small text within a headset. Furthermore, a recent review of studies in cybersickness (i.e., VR-induced motion sickness) show that adjustments to field of view along with a stationary setting (as would be used in a virtual office) can dramatically reduce motion sickness [46]. While the authors are optimistic that motion sickness can be addressed, work in this area of research is ongoing, and we would like to acknowledge that our suggested solution is dependent on solutions to known issues, such as 
sex differences [54] and further study of the phenomenon is required, though not the topic of this paper.

While virtual reality hardware is not yet ready for knowledge workers to don headsets full-time, it may be soon, and this work aims to investigate a future where it is viable. To investigate the potential feasibility of providing the advantages of closed offices via a VR-based office, we conducted a user study to compare several working modalities. We asked participants to complete tasks designed to simulate knowledge work in four different settings: a traditional closed office, a traditional open office (with distractions), a VR-based closed office (with distractions in the real world), and a VR-based open office (with distractions in the VR world). By comparing participants' performance and collecting their preferences, we have gained insight into the trade-offs that these environments offer. While participants performed more tasks more quickly in the traditional closed office than in either open office setting, there was only a small difference between performance in the traditional and VR closed offices. Similarly, while participants had the strongest preference for the traditional closed office, the VR closed office had a similar usability score, and both closed offices were strongly preferred over both open offices. We believe these results show potential that, as VR hardware advances, some of the benefits of closed offices could be brought to open office configurations via VR.

\section{RELATED WORK}

There are several areas of research relevant to this paper. In human-computer interaction (HCI), there has been a large focus on interruption and work performance and there has also been some consideration of flow and how it relates to performance. There is also an abundance of work in virtual reality, much of which focuses on specific domains of work, but there is a dearth of research on using virtual reality to support seated office work.

\section{Interruption and Work Performance}

There has been much attention in HCI paid to the problem of interruption $[1,2,4,26-28,34,38,39,53,66]$ and the general consensus is that distractions hinder productivity. Specifically, people can take over 25 minutes to get back to work after a brief interruption [38], people tend to make more errors in work after even a brief interruption [2,34] and take more time to make decisions [53]. Some studies have also explored the behaviour of people when interrupted and observed that they sometimes delay responding to distractions in order to set up the state of the task they intend to resume later [28], but that interruptions increase task completion time, as it takes time to resume the original task $[1,27,28]$, and this increase is particularly detrimental for knowledge work [26]. While multitasking, interruptions have been found to occur on average 7 times per hour, mostly from email and IM alerts (circa 2007) [28]. A related study found that more task switching led to a higher chance of getting interrupted [38]. In some cases, interrupted work is completed in the same time as non-interrupted work, but with higher stress (mental cost) [39] due to annoyance, frustration, and anxiety interruption $[4,66]$. While there has been significant work on understanding how problematic interruption is, our work adds to this body of literature with a study exploring the use of VR to address interruptions in an open office environment.

\section{Flow and Performance}

Flow is the state of complete immersion in a task, the optimal psychological state when everything comes together for the performer. [11] This affective state of complete absorption has been related to notions of peak performance and peak experience [45]. Several studies found that certain conditions are necessary to reach a state of flow, including balance between challenge and skill [10], the opportunity to learn [11], and selfcontrol within the task [13]. Confidence and concentration have also been shown to facilitate flow [59].

When in flow, people experience genuine enjoyment in their current task, deep focus, time dilation, and a separation from their surroundings [13]. Flow is also associated with higher performance [15] as well as higher quality of performance [59].

In sports, studies suggest that peak performance always appears when in flow [32] and performance is related to flow [56]. Flow was systematically explored in elite sports [57,58] and found to be effectively facilitated by mindfulness-based interventions [50]. Additionally, flow was used as a predictor of success in competition among older athletes [30]. Flow has also been used as a predictor of performance in school curricula $[12,63]$.

In creative work, scientists and artists experience flow frequently in their career. A study showed that most interviewed artists and scientists mentioned flow or similar experiences of separation from conscious activity to doing things automatically [13]. Studies have also explored composing, listening, and playing music in relation to the flow experience $[9,14,17,24,37,42,44,64]$.

In our work, we leverage this vast literature connecting flow and performance by comparing people's ability to get into a state of flow in open office settings and whether the use of virtual reality can help people to achieve this flow state. We hypothesize that higher flow can be achieved in open office settings when VR is used to mitigate distraction.

\section{Virtual Reality and Work}

There are many practical applications of virtual reality, such as $3 \mathrm{D}$ modeling, education, and medicine $[8,23,36,51,55]$. While these could already be considered "work" applications, they are typically specialized uses of the technology to improve some aspect of the work practice, rather than a replacement for more generic desktop work. There have, however, been some attempts to bring $2 \mathrm{D}$ windows into $3 \mathrm{D}$ virtual environments (e.g., [3,5,16]), including commercial applications (e.g., Steam's Virtual Desktop). Other work has explored the use of keyboards for common desk work in offices [20,33,49], using VR for mobile knowledge work [21], and the effects of long-term use of VR in office environments [22].

While the possibility of office work in VR has been explored from a technical perspective, there has been little work exploring the use of this kind of interaction in VR to mitigate distraction in offices. One notable exception is the work by 
McGill et al. [40] which provides evidence that increasing awareness of the real world increases distraction from the VR experience, and suggestions for how much reality to include in VR. Some work has also shown that VR has the potential to help reduce stress, for example by simulating nature [61] or by facilitating meditation [60]. Our work builds on this prior work by specifically targeting office work (programming) and leveraging VR to mitigate distraction and improve flow.

\section{STUDY}

We conducted a laboratory study to investigate the ability for virtual reality to help deal with distraction in open office environments. Specifically, we were interested in the following research questions:

RQ1 Does the use of a VR headset to perform work in an open office environment improve flow?

RQ2 Does the virtual scene rendered in the VR world matter, or will any environment do (even one with an open office simulation)?

RQ3 How close to closed office work can work in VR get?

Our study therefore included four environments to complete work: a traditional open office (baseline), a VR world with a tropical beach background, a VR world with an open office background, and a traditional closed office (the ideal being strived for).

\section{Participants}

We recruited 11 participants in the USA and 24 in Switzerland. We advertised using posters, university mailing lists, and university student job postings. The total pool of 35 participants is nearly balanced across two genders, with 18 identifying as women and 17 identifying as men. Because sex differences in spatial ability are well-documented [35] and have been shown to transfer to VR [54], we aimed to balance male and female participants we selected for our study. The participants had an average age of 27.6 years ( \pm 6.7 ). Among participants, $44 \%$ reported currently working in an open office and $89 \%$ had little or no prior experience with VR.

\section{Location and Technical Setup}

The study was run in a total of three venues: two in the USA and one in Switzerland. In the USA, six sessions took place in a study room of a university library (Figure 1) and five sessions were run in a small conference room at a co-working office. In Switzerland, we ran twenty-four sessions in an office of a university (Figure 2). Between the three venues, the setup was similar: each room had windows and was well-lit. The participant, researcher, and assistant sat together at a large desk, with the researcher positioned near the participant, and the assistant positioned across. The main experimenter was present at all locations to ensure consistency (technical setup, room, etc.) and we carefully trained the assistants to perform distractions in the same way.

Technical Setup. For VR conditions, we used the HTC Vive Pro Eye with the Razer Blade 15 laptop with Nvidia RTX 2080 Max-Q graphics. An external screen was used to project



Figure 1: Open Office setup in a library room in the USA

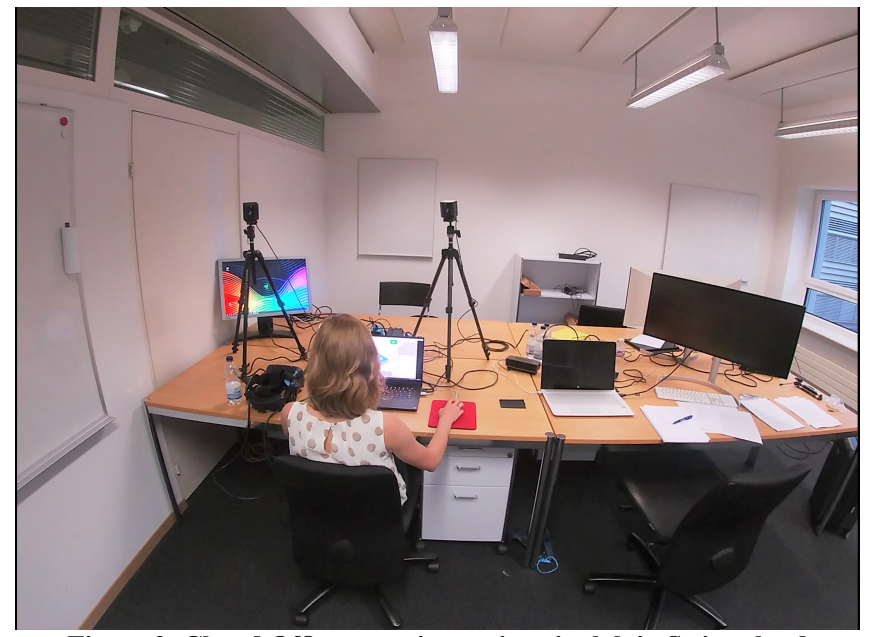

Figure 2: Closed Office setup in a university lab in Switzerland

Lightbot into the virtual desktop. For the non-VR conditions, only the laptop was used. Participants used a computer mouse to perform tasks, both in and out of VR. In VR, this meant that participants could not actually see the mouse and hand. Though this felt somewhat unnatural, in pilot testing we attempted using VR controllers in all conditions (with and without VR), and that proved even less natural. We also limited interaction to mouse-only, so there was no need to reacquire the mouse (e.g., from the keyboard) at any time.

Recordings We ran screen-recording software to document task completion in Lightbot and filmed the experiment with a GoPro camera in order to review interactions later.

\section{Factor: Open vs. Closed Office}

Simulating the open office. The simulation of an open office environment was one of our big puzzles, one which we considered at length. On the one hand, the conditions needed to be reproducible (across task sections and between participants). On the other hand, it had to be believable and thus involve live actors. The resulting setup consisted of scripted interactions between two actors with a backdrop of recorded office sounds, including snippets of conversation, typing, chewing, humming, 
rustling paper, and other office noises. Additionally, for a feeling of authenticity, one researcher and assistant simulated open office distractions. These included holding a simple conversation, occasionally getting up and walking to get something on the other side of the room, and other casual actions native to the office. Apart from a slight change in conversation (to avoid repetition), we practised the distractions to be believable and consistent between conditions and participants. We felt the result is as reasonable a simulation of an open office as could be achieved without recruiting more assistants. We include the study materials, including a copy of the audio track and VR scenes to the Open Science Framework for researchers wishing to reproduce our experiment ${ }^{1}$.

Closed office. The closed office condition was simpler. The participant stayed in the same room, but the researcher and assistant stepped outside for twelve minutes while the participant performed tasks. The participant was instructed to start as soon as the door closes, and to stop when the door reopened on the researcher's return twelve minutes later. This way we modified the environment as little as possible, removing only the people and office noise.

\section{Factor: VR vs. No VR}

For all conditions, the participant was seated at a desk and performed their task using only the computer mouse. For the two non-VR conditions, participants viewed the task on a 15.6" laptop monitor (1080p). For the two VR conditions, participants put on the HTC Vive headset and were allowed a few moments to adjust the fit and focus. The desktop screen with the task was projected on a rectangular surface in the VR environment, so that the participants saw a floating screen before the presented VR background.

\section{Conditions}

We combined these factors into the following four conditions:

Closed office (with no VR): This served as the goal state for comparison against the other conditions. In this condition, the researcher and assistant left the room and gave participants twelve minutes to work through the tasks alone on the laptop (Figure 2).

Open office (with no VR): This served as a baseline to beat. The participant performed tasks on the laptop with the researcher and assistant seated at the same desk (Figure 1, without a headset). An audio track with open-office noises played in the background while the actors simulated office interactions.

Beach VR (open office with a beach VR environment): Same office setup as above. We used the free Hawaiian Beach environment from Steam VR (Figure 3), which features a photorealistic sandy beach, blue skies and palm trees, with wave animations and sounds. The task window was projected in a virtual desktop.

\footnotetext{
$1_{\text {https://osf.io/ajx9s/ }}$

${ }^{2}$ For Figure 4 and Figure 3, the Lightbot overlay was not captured in the screenshots and was reintroduced manually to approximate what the user would have seen. The dotted rectangles indicate the user's approximate field of view.
}

Office VR (open office with a simulated office VR environment): We used a 20-minute recording of a graduate student lab at the university, filmed using a stereo $360^{\circ}$ camera. The virtual environment (Figure 4) features an office setting with four actors who work on computers at their desks and occasionally walk around, engage in conversation, and perform other actions similar to our live open office experience.

We used a within-participants design and counterbalanced these four conditions using a random Latin square.

\section{Task}

For each condition, participants solved tasks in the coding game Lightbot [65], featured in Hour of Code. The fun, progressing challenges make the game a great candidate for inducing flow.The goal of the game is to navigate a little bot on a $2 \mathrm{D}$ board and light up all blue tiles. Rather than typing commands, the player adds a sequence of premade commands to an execution window by clicking on the appropriate tiles. The game gets progressively more challenging with a more complex board, new commands, and the introduction of procedures. With its simple set of commands, the game requires no prior experience and was thus accessible to a wide audience. At the same time, the programming nature of the tasks requires the same type of logical thinking that knowledge workers do on a daily basis. These aspects made Lightbot a good fit for our experiment. After extensive piloting of various tasks, we identified the LB tasks as a good choice that fulfills these criteria. Ideally, we would have chosen tasks with the same difficulty level to have full comparability. The variation of challenge in LB tasks made it harder to compare performance between conditions later on, as we will explain in the results. However, since the concept of flow requires the challenge of the task to match the increase in the participant's skill over time, we identified the LB tasks as a good fit. In addition, the LB tasks assume no prior knowledge of programming, which made them accessible to a broad audience, and the increase in challenge of the LB tasks kept participants interested.

\section{Procedure}

Each study session lasted approximately two hours and had six stages: introduction, four conditions, and wrap-up.

Introduction. The participant was seated and given an overview of the study. They were then asked to complete the first six tasks of level one in Lightbot as a tutorial, in order to familiarize themselves with the program. At this stage, participants were allowed to ask questions if any confusion should occur. This setup had two variations in order to best match the tutorial to the participant's first condition. If the participant's first condition would be without VR, the participant performed the tutorial also without VR, simply using the laptop. If, however, the first condition was in VR, the participant performed the tutorial in VR. For this we used the Steam VR home "Summit Pavilion" environment, which we felt was a neutral option.

Four Conditions. Each participant was randomly assigned one of the four condition orders. For each condition, the participant started with task one of a new level (Level 2 for the first condition, Level 3 for the second, and so on). They would then be given 10-12 minutes to work through the tasks, until 


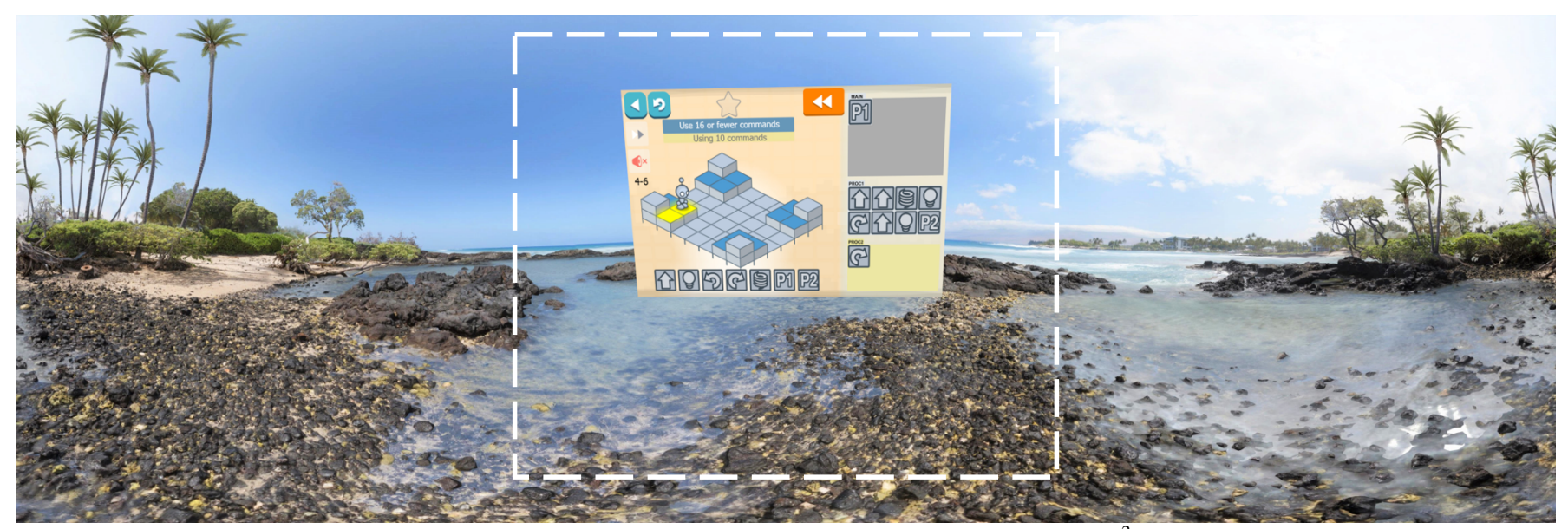

Figure 3: Virtual Hawaiian Beach, an environment created by Steam from photos of Big Island, $\mathrm{HI}^{2}$ and shown here as a panorama.

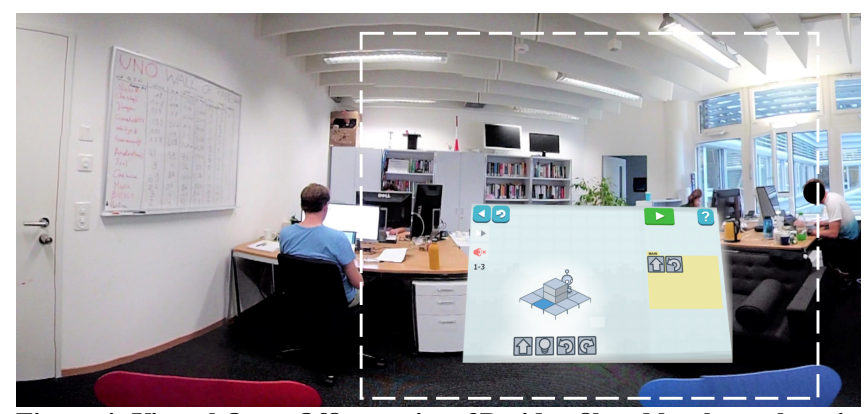

Figure 4: Virtual Open Office setting, 3D video filmed by the authors in an open office (note: cropped from full panorama).

told to stop. In the end, we only counted tasks fully completed before the ten minute mark. However, we allowed participants up to two extra minutes to finish their current task, in order to reduce possible frustration and avoid negatively impacting the flow score. At the end of the condition, the participant was asked to fill out a 36-question survey on the laptop screen to measure their current flow state. Participants were invited to take short breaks if needed.

Wrap-up. Upon concluding the four conditions, the participant was interviewed one-on-one with the researcher for approximately ten minutes to gain an overall understanding of their experience in the experiment and their preference for the conditions. Finally, the participant filled out a demographics questionnaire and was paid.

\section{Measures}

We measured: flow, task completion, and condition preference.

Flow Scale. At the end of each condition, participants filled out a flow scale survey based on Jackson et al.'s Flow Scale Manual [29] to measure the participant's level of flow and engagement during the section. The survey measures flow based on nine major factors, e.g. the challenge-skill balance, loss of self-consciousness, and transformation of time. The questionnaire consisted of 36 statements which the participants rated on a scale of 1 (never) to 5 (always).
Task Completion. For each condition, we counted the number of tasks fully completed before the 10 minute mark. Participants were actually allowed up to 12 minutes to work through a level in case they were stuck on a task, in order to reduce potential frustration and affects on the flow measures. However, we only counted tasks fully completed after ten minutes.

Condition Preference. At the end of the study, participants were interviewed and asked to rank conditions in terms of how well they focused.

\section{RESULTS}

Our observations suggested that, despite our efforts to use a known-to-be-effective progression of programming challenge, the order of Lightbot tasks influenced participants' experience of flow. We therefore performed an exploratory RM-ANOVA with the four environment conditions (closed office, beach VR, office VR, open office) as a within-participants factor that included the order of conditions as a between-participants factor. Our dependent measures were the nine dimensions of the flow scale, the overall flow score, and the number of Lightbot tasks completed within each condition. This analysis is consistent with the guidelines of the Flow Scales Manual [29], and we note that our choice of RM-ANOVA over nonparametric tests is an accepted practice [43]. Our post-hoc analyses used the Bonferroni correction.

\section{Flow Scale}

While we now know that task ordering influenced participants, for completeness we first analyze the results without considering task ordering. In this case there were significant main effects of environment for the following flow scale dimensions: concentration on the task $\left(F_{3,93}=6.8, p<.001, \eta_{p}^{2}=.18\right)$, sense of control $\left(F_{3,93}=4.9, p<.01, \eta_{p}^{2}=.14\right)$, and loss of self-consciousness $\left(F_{3,93}=3.6, p=.02, \eta_{p}^{2}=.10\right)$. The main effect of overall flow was not significant $\left(F_{3,93}=2.5, p=.06\right.$, $\left.\eta_{p}^{2}=.08\right)$.

Post-hoc tests (Figure 5) revealed that the closed office environment was rated higher than open office for the flow dimensions; this difference was significant for concentration on the task and sense of control $(p<.05)$, and not significant 


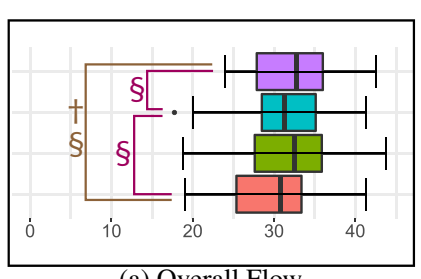

(a) Overall Flow

Closed

BeachVR

OfficeVR

Open

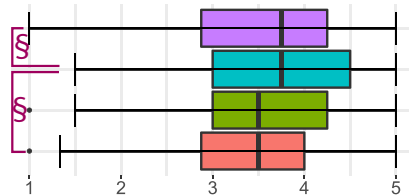

(b) Challenge-Skill Balance



(e) Unambiguous Feedback

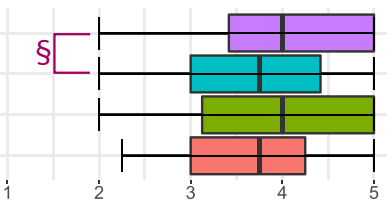

(h) Loss of Selt-Consciousness

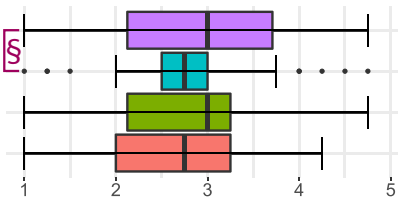

(c) Merging of Action and Awareness

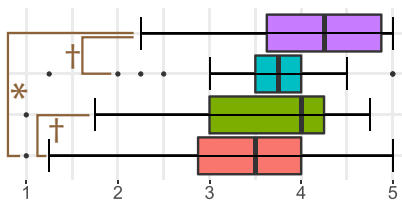

(f) Concentration on the Task at Hand

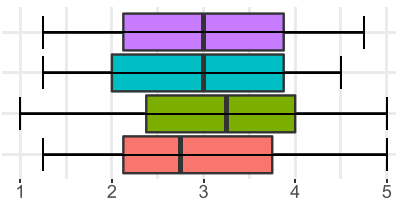

(1) Iranstormation of lime

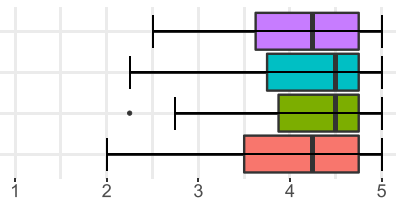

(d) Clear Goals

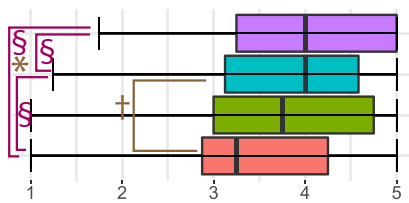

(g) Sense of Control

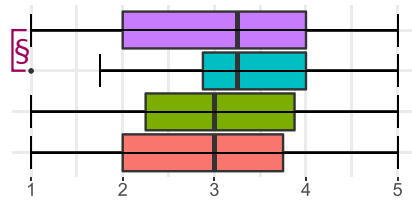

(j) Autotelic Experience

Figure 5: Box plots of the main effects of environment on the overall flow scale and its nine dimensions. Post-hoc pairwise differences are indicated ( $\dagger$ : $p<.08, *: p<.05, * *: p<.01)$ as well as pairs that had a significant interaction with order for some order of conditions ( $\$$ ).

for overall flow $(p=.06)$. While not statistically significant, closed office was also rated higher for concentration on the task than both beach VR $(p=.06)$ and office VR $(p=.06)$ and higher for loss of self-consciousness than beach VR $(p=.06)$. Interestingly, beach VR was also rated higher than open office for sense of control, but this was again not statistically significant $(p=.06)$. Note that these differences, even though not accounting for ordering, show a difference between the closed and open office environments.

\section{Order Effects}

As mentioned, the LightBot tasks influenced participants and so we further analyzed our data with this in mind. While there were no main effects of order itself $\left(F_{3,31}<2.4, p>.09\right)$ there was a significant interaction between environment and order on overall flow score $\left(F_{9,93}=2.8, p<.01, \eta_{p}^{2}=.22\right)$ and on the following dimensions: balance between challenge and skill $\left(F_{9,93}=3.0, p<.01, \eta_{p}^{2}=.23\right)$, merging of action and awareness $\left(F_{9,93}=2.2, p=.03, \eta_{p}^{2}=.18\right)$, sense of control $\left(F_{9,93}=3.1, p<.01, \eta_{p}^{2}=.23\right)$, loss of self-consciousness $\left(F_{9,93}=2.2, p=.03, \eta_{p}^{2}=.18\right)$, and autotelic experience $\left(F_{9,93}=2.4, p=.02, \eta_{p}^{2}=.19\right)$.

Post-hoc analyses on these interactions revealed several differences for participants that saw conditions in the order: open office, office VR, beach VR, closed office. The pattern was that, in this condition, beach VR was rated significantly higher than closed office for balance between challenge and skill $(p=.01)$, merging of action and awareness $(p=.04)$, sense of control $(p=.03)$, autotelic experience $(p<.01)$, and overall flow $(p=.02)$. Beach VR was also rated higher than open office for balance between challenge and skill $(p=.03)$, sense of control $(p=.04)$, and overall flow $(p=.01)$. Beach VR was also rated higher than open office for balance between challenge and skill for participants that saw conditions in the order: beach VR, open office, closed office, office VR $(p=.03)$. When accounting for ordering, beach VR appears to help users achieve flow.

For sense of control and overall flow, participants also rated the closed office condition higher than both beach VR (control: $p<.01$; flow: $p=.02$ ) and open office (control: $p<.001$; flow: $p=.02$ ) when they saw conditions in the order: closed office, beach VR, office VR, open office. Closed office was also rated higher than beach VR for loss of self-consciousness when participants saw conditions in the order: beach VR, open office, closed office, office VR $(p=.03)$. As when not accounting for ordering, the closed office also appears to help users achieve flow for certain orderings.

\section{Summary}

Our findings show a tendency that participants were most in flow in the closed office environment, followed by the beach VR environment, and least in flow in both the office VR and open office environment without VR. However, while the closed office led to significantly higher flow than the open office, the other differences were either not significant or only true for specific orders of presentation of the conditions. While this tendency is not strong statistically, it is fairly consistent, and it is corroborated by our qualitative findings (see below). The qualitative findings also shed light on possible reasons for the lack of clearer differences.

\section{Task Completion (Order Effects)}

There were no main effects of environment $\left(F_{3,72}=0.1, p=\right.$ $\left..94, \eta_{p}^{2}=.01\right)$ or order $\left(F_{1,24}=1.2, p=.33, \eta_{p}^{2}=.13\right)$ on number of tasks completed. There was, however, a significant 


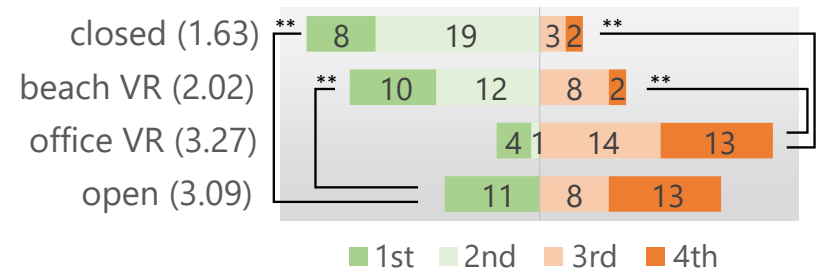

Figure 6: Rankings per condition (Mean Rank from Friedman's ANOVA indicated in brackets). Lines between conditions indicate significant pairwise differences $(* * p<.001)$.

interaction between environment and order $\left(F_{9,72}=9.9, p<\right.$ $\left..001, \eta_{p}^{2}=.55\right)$. Pairwise post-hoc analysis revealed that for every order, the final condition seen by participants always had the fewest tasks completed, and this difference was significant $(p<.05)$ for all but three pairs.

\section{Summary}

It should be noted that Lightbot tasks are not designed to be equivalent in duration; however, the number of tasks completed can be considered an indicator of difficulty. This analysis therefore highlights that difficulty related more to the order of levels in Lightbot than to the conditions in our study. While on the one hand, this could be thought of as a confound in our study (flow depends on the level of challenge) and we encourage the reader to consider our quantitative findings to be exploratory, the fact that the flow dimension analysis did not have this same last-condition dominance lends more weight to the observed effects of environment on flow. Specifically, in the flow analysis, the environment condition accounted for more of the variance and participants consistently rated closed office highest, followed by beach VR, then office VR and open office; conversely, in the task completion analysis, the Lightbot levels were a better predictor of difficulty.

\section{INTERVIEW FINDINGS}

Based on the transcripts of the semi-structured interviews with all participants, we derived participants' preference ranking for the four conditions and two authors performed a thematic analysis [6] and independently coded the statements of all study participants. Both authors identified emerging themes in the data, and discussed and merged them iteratively. In the following, we report on the results and the main themes and observations.

\section{Preferred Conditions}

As part of their post-experiment interview, participants were asked to rank the four conditions according to preference. We received a complete ordered ranking for 30 participants, partial rankings for two participants who considered two conditions equal, and no ranking for one participant who felt that all conditions were equal. We performed a Friedman's ANOVA to compare ranks (Figure 6), which was significant $\left(\chi^{2}(3)=\right.$ $37.5, p<.001)$. Post-hoc tests revealed that both closed office and beach VR were ranked higher than office VR and open office environments (all pairwise differences $p<.001$ ). There was no significant difference between closed office and beach $\operatorname{VR}(p=.27)$ nor between open office and office $\operatorname{VR}(p=.53)$.
The high rankings of the closed office and the beach condition were supported by many positive comments. For instance, participants stated that the closed office "was silent and I could focus the best" (Z14) and "it was very easy to stay focused" $(S 10)$ in it, and for the beach VR condition that it was their "favorite" (S12) and that in "the beach VR [they] could do a lot more, like fully concentrate" (Z21).

These positive comments contrast with the mainly negative comments from the open office conditions. For instance, for the open office without VR one participant stated that "that was my least favorite because I could hear you clearly, and I didn't like it. As well, I was like... I was trying to focus, but then I was also listening to you too, so that's why I don't like it." (Z05). Another participant elaborated, saying:

"I mean it's really night and day [between the closed and open office]. At some moment in time [in the open office setting], one of you shared a meme with the other person. Immediately, became more interested in that than what I was doing I was like 'I want to see too.' That's why I leave the office.. because.. I just need focus." (SO3)

The lack of difference between the closed office and beach VR condition is also supported by the similarity in participants' comments about these two environments. For instance, participants commented for both conditions that it was easy to stay focused in them because "there just weren't any distractions" (S10) in the closed office and that they were "not really aware of anything else in the beach" (SO2). Similarly, the comments on the open office and open office VR conditions overlapped often, since they focused a lot on the experienced distractions, such as the background noise that "was the worst" (Z09) as someone stated or another one saying "when there were background noise or talking, that's where my mind was sometimes going back and forth" (Z21). This contrast in experiencing distractions or not further illustrates the preference of the closed office and beach VR conditions to the open office and open office VR condition.

The closed office and beach VR conditions were both significantly preferred over the open office and open office VR conditions.

\section{Distractions}

Participants mentioned an array of impediments to achieving focus and flow. In general, they differentiated between auditory distractions, such as people talking or chewing nearby, and visual distractions, such as people walking by. There are, however, also other more subtle sources for distractions, such as feeling of someone "looking at you" (SO2) or "too many things that [you] can do" (ZO2) in the place you are in. Overall, auditory distractions were mentioned the most and were in many cases also perceived as more distracting than visual ones: "in the end, I will look at my screen and if I am focused, I will ignore what is happening outside of my screen so to say, but the noise is harder to cancel out I think." (Z2O). What was considered an auditory distraction depends on factors, such as the familiarity of the people talking or the content of the conversation:

"It depends. If it's just background noise then it's fine. If it's 
really a conversation that I may be even a bit interested in, like your cat. I was like "what, someone is talking about cats?!". So I automatically pay attention to that so that's distracting. If it's just background noise, it's fine. So in terms of distraction, I would say interesting conversations are the worst distracting, [more] than movement and than background noise." (ZO3)

At the same time, how distracting something is perceived is very individual and task dependent. While some prefer "to be in a quiet environment alone" (Z19), for others it can be "too quiet" (Z07) or "so silent [in the closed office that they] didn't like it very much" (Z06). Several participants stated that they like working in environments where there is white background noise, such as a coffee shop or library, or they listen to music, mostly without lyrics, such as "soundtracks from video games" (Z13). Such constant noise can help to block out distractions: "I usually always listen to music .. then I can't hear others making noise with their papers and stuff" (ZO2). Similarly to the individual differences, the type of task has an influence on the perception of distractions and what works to block them out, as for instance one participant stated "it really depends on the task. But I either seek out complete silent or coffee shop where there's like background noise" (Z09). Participants often mentioned adjusting the music and listening behavior to the task: "When I study for an exam, I don't listen to music. But when I write a report for a laboratory, or stuff like that, then there's always music" (ZO7). Finally, the perception of certain sources as distractions can also change over time and the tolerance threshold can increase:

"I didn't use to, but I think I've gotten use to it. Now I can focus quite well. I still prefer no one around me. When I was at uni I would always go to the silent bits of the library, where no one is talking, and even the smallest distraction would really annoy me. But having work now, I think it's much easier to focus in an open office, just block it out." (Z24)

Furthermore, the effect of the distraction varies strongly, including people losing focus and concentration, their "mind ... wandering off" (Z21) and they switch away from their work task: "I have very good peripheral vision. So, I have a hard time not looking up or leaning towards an object, therefore distracting myself" (S12). Participants also mentioned getting frustrated and annoyed, which in turn impedes them from getting into flow and decreases their focus at work.

Distractions are often of auditory or visual nature and their perception and effect varies by individual and task.

\section{(Beach) VR Effects}

In our interviews, participants commented often on the effects of the VR, in particular the beach VR condition. Most participants noted that the beach VR helped them focus, to "wash away" (Z07) and reduce external interruptions or distractions: "I was more focused with the beach I would say. I was able to focus ... I didn't even notice you guys were there" (S03), or "suddenly [in the closed office] I caught myself looking around a bit, and just looking at the office, and just getting annoyed by the computer sounds, like the air ventilation of the computer, and that was all gone in the beach setting." (ZO7). By placing users in a different environment, it also reduced the options for self-distraction that exist even in quiet rooms:

"It would take me away from my reasons for not liking to study or work at home, because it is a home environment, and I always [think]... 'I'm going to go to the bathroom. Oh, my bed is right there. I'm going to sleep,' which is not a good thing. So, if I were to wear a headset, it would at least transport me away from my bedroom, even though I'm in my bedroom." (S12)

Additionally, participants valued the feelings and mood the beach environment induced, such as a feeling of calm and being relaxed and commented on the importance of the kind of VR environment used: "the beach one. It just affected my mood, sitting around the beach, and some nature environment feeling. And then, this way, I could concentrate better. The VR environment is very important" (Z21). Several participants also explicitly mentioned the positive effect of the feeling of not being watched in the beach environment compared to the others: "because I felt that I was [alone] and nobody was watching me and it was quite relaxing to see the beach." (Z15). Finally, one participant also commented on the ability of the VR environment to foster creativity since in the right environment "your brain starts to think in other way[s]" (Z23).

However, three participants also explicitly mentioned that the beach environment itself can be distracting, since "you are not in a working environment, that makes it less focused" (Z14) and the beach "was just not the place to be on the computer" (Z17). A further negative effect mentioned with respect to the VR is the social detachment, since the VR "detach[es you] so much from everybody else socially" (Z13) while it is often good to have some people around for work for social reasons.

VR can reduce distractions and increase well-being for many, but it matters a lot which VR environment is used.

\section{VR Usage Experience}

When asked whether they would consider using VR for short periods each day, many participants commented that they would for specific tasks, but that, especially due to its comfort, they wouldn't wear it for all day and that it also depends on which other environments are available. One participant, for instance, mentioned

"I mean, like for a few minutes, 20 minutes. That would be no problem, but I think if I had to wear this the whole day, it would be too heavy. If it would advance in a technical way, like weigh less and high resolution, I could imagine wearing it all day. Yeah, if it's not physically a disturbance." (Z2O)

Overall, many participants stated that the VR was comfortable enough for short periods of time, however, several issues were also raised about the experience wearing and using the VR and its comfort, in particular with respect to its weight, its heat, the blurriness at the edges, the eyes hurting after a while and for two participants also the dizziness they experienced in the VR condition.

VR is an option for most but only for shorter time periods, especially due to comfort concerns. 


\section{Varying Task Difficulty}

Independent of the conditions, we also asked participants about their experience performing the given tasks. While many enjoyed the tasks, stating, for instance, that they "liked [them] quite much" (Z16) and that "it was fun" (SO3), most participants commented on the varying level of difficulty of the tasks and that the later ones were more difficult. While some considered the increasing difficulty level a fun and engaging challenge, "The beginning I felt like, a little more bored, but the later ones I was definitely very engaged. So the more challenging tests were more engaging." (S10), others even perceived it as frustrating and annoying, for instance, "I was into it at first, but then when I got to the ones that I couldn't do, I just was like, 'Let's just keep it going on repeat until the 12 minutes are over." (Z24), or "Except for the last one, it was fun actually. I liked them. Just the last one was really frustrating." (Z06).

Our study tasks were generally well-suited for keeping participants engaged, however, participants perceived a significant increase in their level of difficulty.

\section{DISCUSSION}

The goal of our study was to examine whether we can create a VR environment for an open office environment that provides similar benefits to that of a closed office for work. The qualitative and quantitative results of our study show that this idea has potential. Our qualitative results show that participants have a clear preference for the beach VR over the open office and open office VR conditions, as seen in the interviews from both countries. Our exploratory quantitative analysis provides evidence that further supports this observation, yet task ordering and task difficulty clearly had an effect on results. In the following, we will discuss the implications of our research.

\section{Overcoming Workspace Limitations}

Extensive planning goes into the creation of today's workplaces to best support knowledge workers. At the same time, companies have limited resources in terms of space and money, and despite the knowledge that open office workplaces can reduce workers' satisfaction and productivity, many companies have or are moving towards open office work environments [7]. One way that some companies are trying to overcome the increased number of distractions in an open office environment is by providing noise cancelling headphones or mounting white noise speakers in the office. Yet, since the distractions that knowledge workers experience in an open office are not just auditory, these solutions only partially address the problem. The results of our study show that we might be able to take advantage of VR, at least for certain periods of time, and "transport" the knowledge worker to a more desired work environment, regardless of physical space limitations. With the recent advances in VR technology there is a huge potential to better support knowledge workers in the future, especially where closed offices are not a viable solution. Even outside of the office, such as the home or the airport, where the distractions are of a different nature, we believe this approach could improve focus.
Tailoring the Environment to the Individual and Task

The data of our study shows that the details of an ideal work environment are dependent on both the individual and the task. While some participants prefer complete silence in a closed space, others prefer a coffee shop, or adjust the type of music they listen to based on the task they are working on. Similarly, while most participants in our study enjoyed working in the beach VR environment and several even preferred it to the closed office, some participants did not like the the mixing of work with a beach environment which they considered more for leisure. Fortunately, VR is highly customizable and one can think up an infinite number of environments to work in. Users of a VR office could tailor the work setting to their needs and preferences, even changing environments to suit their task. For instance, when knowledge workers have to perform rote work, they can choose a more stimulating environment, for cognitively demanding assignments possibly a more quiet and calm environment, and for creative tasks an environment that fosters creativity, e.g. by increasing the blue light portion [47]. In the future, we might also be able to automatically adjust the environment to the individual on a moment-by-moment basis by using biometrics (e.g., [52]).

\section{One Environment Does Not Fit All}

While VR has great potential to help knowledge workers achieve flow, we are not suggesting that they work in VR all day long. Today's headsets have clear limitations, especially in terms of comfort, screen resolution, and weight. Additionally, the headset can lead to social detachment, which participants mentioned several times, and causes challenges in interacting with the real world. For instance, based on our experience using the VR extensively for this study, drinking coffee while working in VR is a challenge at best, if not outright dangerous. Therefore, the goal is not to completely replace the usual work environment with VR environments, but to provide the opportunity for transforming the work setting to achieve the right state of mind. Future studies should examine for which situations a VR environment is best suited and how well it can integrate into the usual day of a knowledge worker's life. Furthermore, we must consider the macro effects that such technology would impose on the workplace. Though virtual reality could allow participants to escape to an isolated world and fully focus on their work, it would be at a trade-off with the collaborative factors of the open office. It would be interesting to study how VR technology would play out on the social dynamics of the workplace.

\section{Choosing Study Tasks}

The goal for our study was to examine the effect of different environments while performing work tasks. Therefore, we tried to compile study tasks that met many constraints. They had to be engaging and challenging, yet not too difficult; flow inducing, but not too time-consuming; comparable with each other, yet doable by a broad audience. At the same time, these tasks had to comply within current VR technology and its limitations, such as the limited resolution of headsets and the difficulty of VR keyboard input. After several iterations and extensive piloting, we chose the Lightbot tasks, programming tasks that can be solved by people even without programming 
experience. However, despite the program's organization into levels of increasing complexity which slowly builds upon previous levels, users felt that the difficulty varied dramatically for certain levels, as their comments have shown. In general, it is challenging to identify a good set of comparable work tasks that fit the requirements of such studies. Identifying and sharing such tasks with the research community could significantly facilitate research in this domain and its generalizability. Thus, while our quantitative results are less generalizable due to the ordering effects, we believe that they provide initial evidence that illustrates the potential of VR work environments. Further studies are needed, either with a more comparable set of study tasks or a large number of participants to examine the generalizability.

\section{Our Mixed-Methods Approach}

Given the varying difficulty of the study tasks, we designed the study to use a mixed methods approach to evaluate the viability of using VR in realistic work settings. As with any study, there are tradeoffs that come with study design choices. In our study, we traded off some control on exact task difficulty and its comparability for the realism of an office setting with realistic programming-like tasks. By using a mixed methods approach, we were able to triangulate the qualitative with the quantitative findings. Note that our claims stem from a mixed methods approach and are not meant to reflect a more traditional hypothesis test (where the importance of p-values are paramount), but instead we take the approach of reporting exact p-values, eta squared effect sizes, using different symbols for different thresholds in our figures, and considering these all in the context of qualitative findings from the interviews with participants. We believe that this triangulation and the qualitative analysis is actually one of the strengths of our research and it is an important step in a larger body of work that can further triangulate these findings, perhaps with a larger sample or a longitudinal study.

\section{THREATS AND LIMITATIONS}

One threat to the validity of our study is the short duration of conditions. To avoid possible exhaustion, we designed our study to fit in two hours. With the time required for setup, transitions, and wrap-up, this gave participants only twelve minutes for Lightbot in each of the four conditions. Whether this is truly enough time to get into the flow state is hard to say, and is highly variable by individual. This may be the reason why we did not see significant differences in flow measures between conditions.

Furthermore, our simulated open office environment and the Lightbot tasks does not claim to accurately represent the daily environment and tasks of knowledge workers. To generalize our findings to the industry we would need to run longitudinal studies with knowledge workers in a real open office.

Some extraneous variables were particularly problematic in our experiment. Most of our participants were first-time VR users, and the novelty and excitement of using the VR may potentially affect the results more than our independent variables. Furthermore, the challenge of certain Lightbot levels (particularly in conditions two and four) required significantly more thought and time for completing tasks. As a result, performance was effected more by the task rather than by the condition. Although we tried to control for novelty and level difficulty through order randomization, the effect of these variables was so diverse between participants, that it introduced high noise levels. A longer-term field study would likely reduce these limitations. Another extraneous variable is the auditory factor, which is a natural part of the VR environment. Both VR environments included sound (beach waves or office noises) for an immersive experience. It would be interesting to explore how much participants are affected by the auditory factors as opposed to the visual.

\section{CONCLUSION}

Is VR the future solution for bringing the isolation of a closedoffice space into a busy open office? In a lab study with 35 participants, we examined the effects of virtual and traditional work environments on knowledge workers while performing work-related tasks. The qualitative and exploratory quantitative results show that the closed office and the beach VR are similarly good according to users' ranking in reducing distractions and inducing flow, and that these two environments are preferred over the non-VR open office and VR open office environments. Further studies need to be run to examine the generalizability of our quantitative results.

Overall, these results indicate the potential that VR environments have to help knowledge workers achieve flow and stay calm and focused even in loud open office work settings. At the same time, the results open up new opportunities for research. Since the preferred work environment is highly individual, we need to examine which environment is best suited for which situation. Considering the high customizability of VR and the nearly infinite possibilities for environments, we may be able to tailor the VR work setting to individual preferences, the task, and possibly even the current mental state of the knowledge worker to provide the best experience in every moment. At the same time, we need to explore how this technology will alter workplace dynamics and social interactions among knowledge workers of the future, due to the current limitations of the technology.

\section{ACKNOWLEDGEMENTS}

The authors would like to thank all study participants and assistants involved. We also thank the Natural Sciences and Engineering Research Council of Canada (NSERC), the Saskatchewan-Waterloo Games User Research (SWaGUR) program, and the Ontario Ministry of Research, Innovation and Science for funding. We would also like to thank Willamette University for providing a space to run our study.

\section{REFERENCES}

[1] Erik M. Altmann and J. Gregory Trafton. 2002. Memory for goals: an activation-based model. Cognitive Science 26, 1 (Feb 2002), 39-83. DOI :

http://dx.doi .org/10.1016/S0364-0213(01)00058-1

[2] Erik M. Altmann, J. Gregory Trafton, and David Z. Hambrick. 2014. Momentary interruptions can derail the train of thought. Journal of Experimental Psychology: 
General 143, 1 (2014), 215-226. DOI :

http://dx.doi.org/10.1037/a0030986

[3] Carlos Andujar and Ferran Argelaguet. 2007. Virtual pads: Decoupling motor space and visual space for flexible manipulation of 2D windows within VEs. In 2007 IEEE Symposium on 3D User Interfaces. IEEE, 99-106. DOI:

http://dx.doi.org/10.1109/3DUI.2007.340781

[4] Brian P. Bailey and Joseph A. Konstan. 2006. On the need for attention-aware systems: Measuring effects of interruption on task performance, error rate, and affective state. Computers in Human Behavior 22, 4 (Jul 2006), 685-708. DOI :

http://dx.doi.org/10.1016/j.chb.2005.12.009

[5] Mark Billinghurst and Thad Starner. 1999. Wearable Devices: New Ways to Manage Information. In Computer, Vol. 32. IEEE, New York, NY, USA, 57-64. DOI: http://dx.doi .org/10.1109/2.738305

[6] Virginia Braun and Victoria Clarke. 2006. Using Thematic Analysis in Psychology. Qualitative research in psychology 3 (Jan 2006), 77-101. DOI :

http://dx.doi.org/10.1191/1478088706qp0630a

[7] Aoife Brennan, Jasdeep S. Chugh, and Theresa Kline. 2002. Traditional versus open office design: A longitudinal field study. Environment and Behavior 34, 3 (2002), 279-299. DOI:

http://dx.doi.org/10.1177/0013916502034003001

[8] Jurgen Broeren, Martin Rydmark, and Katharina Stibrant Sunnerhagen. 2004. Virtual reality and haptics as a training device for movement rehabilitation after stroke: a single-case study. Archives of physical medicine and rehabilitation 85, 8 (2004), 1247-1250. DOI :

http://dx.doi.org/10.1016/j.apmr.2003.09.020

[9] Alice Chirico, Silvia Serino, Pietro Cipresso, Andrea Gaggioli, and Giuseppe Riva. 2015. When music "flows". State and trait in musical performance, composition and listening: a systematic review. Frontiers in psychology 6 (2015), 906. DOI : http://dx.doi.org/10.3389/fpsyg.2015.00906

[10] Mihaly Csikszentmihalyi and Isabella Csikszentmihalyi. 1988. Introduction to Part IV. In Optimal Experience: Psychological Studies of Flow in Consciousness, Mihaly Csikszentmihalyi and Isabella Selega Csikszentmihalyi (Eds.). Cambridge University Press, 251-265.

[11] Mihaly Csikszentmihalyi and Judith LeFevre. 1989. Optimal experience in work and leisure. Journal of Personality and Social Psychology 56, 5 (1989), 815-822. DOI :

http://dx.doi.org/10.1037/0022-3514.56.5.815

[12] Mihaly Csikszentmihalyi, Kevin Rathunde, and Samuel Whalen. 1997. Talented teenagers: The roots of success and failure. Cambridge University Press.
[13] Mihaly Czikszentmihalyi. 1990. Flow: The psychology of optimal experience. New York: Harper \& Row.

[14] Frank M. Diaz. 2013. Mindfulness, attention, and flow during music listening: An empirical investigation.

Psychology of Music 41, 1 (2013), 42-58. DOI:

http://dx.doi.org/10.1177/0305735611415144

[15] Robert Eisenberger, Jason R. Jones, Florence Stinglhamber, Linda Shanock, and Amanda T. Randall. 2005. Flow experiences at work: for high need achievers alone? Journal of Organizational Behavior 26, 7 (Nov 2005), 755-775. DOI:

http://dx.doi.org/10.1002/job. 337

[16] Steven Feiner, Blair MacIntyre, Marcus Haupt, and Eliot Solomon. 1993. Windows on the World: 2D Windows for 3D Augmented Reality. In Proceedings of the 6th Annual ACM Symposium on User Interface Software and Technology (UIST '93). ACM, New York, NY, USA, 145-155. DOI :

http://dx.doi.org/10.1145/168642.168657

[17] Clive J. Fullagar, Patrick A. Knight, and Heather S. Sovern. 2013. Challenge/skill balance, flow, and performance anxiety. Applied Psychology 62, 2 (2013), 236-259. DOI:

http://dx.doi.org/10.1111/j.1464-0597.2012.00494.x

[18] Paul Graham. 2009. Maker's Schedue, Manager's Schedule. (2009).

http://www. paulgraham. com/makersschedule.html.

[19] Cameron Grout, William Rogers, Mark Apperley, and Steve Jones. 2015. Reading text in an immersive head-mounted display: An investigation into displaying desktop interfaces in a 3D virtual environment. In Proceedings of the 15th New Zealand Conference on Human-Computer Interaction (CHINZ 2015). ACM, New York, NY, USA, 9-16. DOI : http://dx.doi.org/10.1145/2808047.2808055

[20] Jens Grubert, Eyal Ofek, Michel Pahud, and Per Ola Kristensson. 2019. The Office of the Future: Virtual, Portable, and Global. IEEE Computer Graphics \& Applications 38, 6 (January 2019), 125-133. DOI: http://dx.doi.org/10.1109/MCG.2018.2875609

[21] Jens Grubert, Lukas Witzani, Eyal Ofek, Michel Pahud, Matthias Kranz, and Per Ola Kristensson. 2018. Text entry in immersive head-mounted display-based virtual reality using standard keyboards. In 2018 IEEE Conference on Virtual Reality and $3 D$ User Interfaces (VR). IEEE, 159-166. DOI : http://dx.doi.org/10.1109/VR. 2018.8446059

[22] Jie Guo, Dongdong Weng, Zhenliang Zhang, Haiyan Jiang, Yue Liu, Yongtian Wang, and Henry Been-Lirn Duh. 2019. Mixed Reality Office System Based on Maslow's Hierarchy of Needs: Towards the Long-Term Immersion in Virtual Environments. In 2019 IEEE International Symposium on Mixed and Augmented Reality (ISMAR). 224-235. DOI : http://dx.doi.org/10.1109/ISMAR. 2019.00019 
[23] Josef Haik, Ariel Tessone, Ayala Nota, David Mendes, Liat Raz, Oren Goldan, Elli Regev, Eyal Winkler, Elisheva Mor, Arie Orenstein, and Ilana Hollombe. 2006. The use of video capture virtual reality in burn rehabilitation: the possibilities. Journal of Burn Care \& Research 27, 2 (2006), 195-197. DOI :

http://dx.doi.org/10.1097/01.BCR.0000200890.34084.76

[24] Emma Hart and Zelda Di Blasi. 2015. Combined flow in musical jam sessions: A pilot qualitative study. Psychology of Music 43, 2 (2015), 275-290. DOI : http://dx.doi.org/10.1177/0305735613502374

[25] Veronica Hollinger. 1990. Cybernetic deconstructions: Cyberpunk and postmodernism. Mosaic 23, 2 (1990), 29.

[26] Shamsi T. Iqbal and Brian P. Bailey. 2005. Investigating the effectiveness of mental workload as a predictor of opportune moments for interruption. In $\mathrm{CHI}$ '05 Extended Abstracts on Human Factors in Computing Systems (CHI EA '05). ACM, New York, NY, USA, 1489-1492. DOI:

http://dx.doi.org/10.1145/1056808.1056948

[27] Shamsi T. Iqbal and Brian P. Bailey. 2006. Leveraging characteristics of task structure to predict the cost of interruption. In Proceedings of the SIGCHI Conference on Human Factors in Computing Systems (CHI '06). Association for Computing Machinery, New York, NY, USA, 741-750. DOI :

http://dx.doi.org/10.1145/1124772.1124882

[28] Shamsi T. Iqbal and Eric Horvitz. 2007. Disruption and recovery of computing tasks: Field study, analysis, and directions. In Proceedings of the SIGCHI conference on Human factors in computing systems (CHI '07).

Association for Computing Machinery, New York, NY, USA, 677-686. DOI :

http://dx.doi.org/10.1145/1240624.1240730

[29] Sue Jackson, Bob Eklund, and Andrew Martin. 2010. The Flow Scales Manual. California: Mind Garden Inc.

[30] Susan A. Jackson, Stephen K. Ford, Jay C. Kimiecik, and Herbert W. Marsh. 1998. Psychological correlates of flow in sport. Journal of Sport and Exercise Psychology 20, 4 (1998), 358-378. DOI :

http://dx.doi.org/10.1123/jsep.20.4.358

[31] Hyun K Kim, Jaehyun Park, Yeongcheol Choi, and Mungyeong Choe. 2018. Virtual reality sickness questionnaire (VRSQ): Motion sickness measurement index in a virtual reality environment. Applied ergonomics 69 (2018), 66-73. DOI :

http://dx. doi .org/10.1016/j . apergo.2017.12.016

[32] Jay C Kimiecik and Susan A Jackson. 2002. Optimal experience in sport: A flow perspective. In Advances in sport psychology (2 ed.), Thelma S. Horn (Ed.). Human Kinetics, 501-527.

[33] Pascal Knierim, Valentin Schwind, Anna Maria Feit, Florian Nieuwenhuizen, and Niels Henze. 2018. Physical Keyboards in Virtual Reality: Analysis of
Typing Performance and Effects of Avatar Hands. In Proceedings of the 2018 CHI Conference on Human Factors in Computing Systems (CHI '18). ACM, New York, NY, USA, Article 345, 9 pages. DOI : http://dx.doi.org/10.1145/3173574.3173919

[34] Kara A Latorella. 1998. Effects of modality on interrupted flight deck performance: Implications for data link. In Proceedings of the Human Factors and Ergonomics Society Annual Meeting, Vol. 42. SAGE Publications, 87-91. DOI: http://dx.doi .org/10.1177/154193129804200120

[35] Marcia C. Linn and Anne C. Petersen. 1985. Emergence and Characterization of Sex Differences in Spatial Ability: A Meta-Analysis. Child Development 56, 6 (1985), 1479-1498. DOI :

http://dx.doi.org/10.2307/1130467

[36] Benjamin Lok, Richard E Ferdig, Andrew Raij, Kyle Johnsen, Robert Dickerson, Jade Coutts, Amy Stevens, and D Scott Lind. 2006. Applying virtual reality in medical communication education: current findings and potential teaching and learning benefits of immersive virtual patients. Virtual Reality 10, 3-4 (2006), 185-195. DOI : http://dx.doi.org/10.1007/s10055-006-0037-3

[37] Raymond MacDonald, Charles Byrne, and Lana Carlton. 2006. Creativity and flow in musical composition: An empirical investigation. Psychology of Music 34, 3 (2006), 292-306. DOI :

http://dx.doi.org/10.1177/0305735606064838

[38] Gloria Mark, Victor M Gonzalez, and Justin Harris. 2005. No task left behind?. In Proceedings of the SIGCHI conference on Human factors in computing systems - CHI '05. ACM Press, New York, New York, USA, 321. DOI :

http://dx.doi.org/10.1145/1054972.1055017

[39] Gloria Mark, Daniela Gudith, and Ulrich Klocke. 2008. The cost of interrupted work. In Proceeding of the twenty-sixth annual CHI conference on Human factors in computing systems - CHI '08. ACM Press, New York, New York, USA, 107. DOI:

http://dx.doi.org/10.1145/1357054.1357072

[40] Mark McGill, Daniel Boland, Roderick Murray-Smith, and Stephen Brewster. 2015. A Dose of Reality: Overcoming Usability Challenges in VR Head-Mounted Displays. In Proceedings of the 33rd Annual ACM Conference on Human Factors in Computing Systems (CHI '15). ACM, New York, NY, USA, 2143-2152. DOI: http://dx.doi.org/10.1145/2702123.2702382

[41] Cal Newport. 2016. Deep work: Rules for focused success in a distracted world. Hachette UK.

[42] Luc Nijs, Pieter Coussement, Bart Moens, Denis Amelinck, Micheline Lesaffre, and Marc Leman. 2012. Interacting with the Music Paint Machine: Relating the constructs of flow experience and presence. Interacting with Computers 24, 4 (2012), 237-250. DOI : http://dx.doi.org/10.1016/j.intcom.2012.05.002 
[43] Geoff Norman. 2010. Likert scales, levels of measurement and the "laws" of statistics. (2010), 625-632. Issue 15. DOI :

http://dx.doi.org/10.1007/s10459-010-9222-y

[44] Susan O'Neill. 1999. Flow theory and the development of musical performance skills. Bulletin of the council for Research in Music Education (1999), 129-134.

[45] Gayle Privette. 1983. Peak experience, peak performance, and flow: A comparative analysis of positive human experiences. Journal of Personality and Social Psychology 45, 6 (1983), 1361-1368. DOI : http://dx.doi.org/10.1037/0022-3514.45.6.1361

[46] Lisa Rebenitsch and Charles Owen. 2016. Review on cybersickness in applications and visual displays. Virtual Reality 20, 2 (01 Jun 2016), 101-125. DOI: http://dx.doi .org/10.1007/s10055-016-0285-9

[47] Gloria Mark Paul Johns Saeed Abdullah, Mary Czerwinski. 2016. Shining (Blue) Light on Creative Ability. In UbiComp' 16 Proceedings of the 2016 ACM International Joint Conference on Pervasive and Ubiquitous Computing. ACM, New York, NY, USA, 793-804. DOI :

http://dx.doi.org/10.1145/502390.502404

[48] Owen Schaffer. 2013. Crafting fun user experiences: A method to facilitate flow. Human Factors International (2013).

[49] Daniel Schneider, Alexander Otte, Travis Gesslein, Philipp Gagel, Bastian Kuth, Mohamad Shahm Damlakhi, Oliver Dietz, Eyal Ofek, Michel Pahud, Per Ola Kristensson, Jörg Müller, and Jens Grubert. 2019. ReconViguRation: Reconfiguring Physical Keyboards in Virtual Reality. IEEE Transactions on Visualization and Computer Graphics 25, 11 (October 2019), 3190-3201. DOI :

http://dx.doi.org/10.1109/TVCG. 2019.2932239

[50] John Scott-Hamilton, Nicola S. Schutte, and Rhonda F. Brown. 2016. Effects of a mindfulness intervention on sports-anxiety, pessimism, and flow in competitive cyclists. Applied Psychology: Health and Well-Being 8, 1 (2016), 85-103. DOI :

http://dx.doi .org/10.1111/aphw. 12063

[51] Sam R Sharar, William Miller, Aubriana Teeley, Maryam Soltani, Hunter G Hoffman, Mark P Jensen, and David R Patterson. 2008. Applications of virtual reality for pain management in burn-injured patients. Expert review of neurotherapeutics 8, 11 (2008), 1667-1674. DOI :

http://dx.doi.org/10.1586/14737175.8.11.1667

[52] Jaime Snyder, Mark Matthews, Jacqueline Chien, Pamara F. Chang, Emily Sun, Saeed Abdullah, and Geri Gay. 2015. MoodLight: Exploring Personal and Social Implications of Ambient Display of Biosensor Data. In Proceedings of the 18th ACM Conference on Computer Supported Cooperative Work \& Social Computing (CSCW'15). 143-153.

http://doi .acm.org/10.1145/2675133.2675191
[53] Cheri Speier, Joseph S. Valacich, and Iris Vessey. 1999. The influence of task interruption on individual decision making: An information overload perspective. Decision Sciences 30, 2 (1999), 337-360. DOI:

http://dx.doi.org/10.1111/j.1540-5915.1999.tb01613.x

[54] Kay M. Stanney, Kelly S. Hale, Isabelina Nahmens, and Robert S. Kennedy. 2003. What to Expect from Immersive Virtual Environment Exposure: Influences of Gender, Body Mass Index, and Past Experience. Human Factors 45, 3 (2003), 504-520. DOI :

http://dx.doi.org/10.1518/hfes.45.3.504.27254

[55] Hemant Bhaskar Surale, Aakar Gupta, Mark Hancock, and Daniel Vogel. 2019. TabletInVR: Exploring the Design Space for Using a Multi-Touch Tablet in Virtual Reality. In Proceedings of the 2019 CHI Conference on Human Factors in Computing Systems (CHI '19). ACM, New York, NY, USA, Article 13, 13 pages. DOI:

http://dx.doi.org/10.1145/3290605.3300243

[56] Christian Swann, Lee Crust, Patricia Jackman, Stewart A Vella, Mark S Allen, and Richard Keegan. 2017. Psychological states underlying excellent performance in sport: toward an integrated model of flow and clutch states. Journal of Applied Sport Psychology 29, 4 (2017), 375-401. DOI:

http://dx.doi.org/10.1080/10413200.2016.1272650

[57] Christian Swann, Richard Keegan, Lee Crust, and David Piggott. 2016. Psychological states underlying excellent performance in professional golfers:"Letting it happen" vs. "making it happen". Psychology of Sport and Exercise 23 (2016), 101-113. DOI:

http://dx.doi.org/10.1016/j.psychsport. 2015.10.008

[58] Christian Swann, Richard J. Keegan, David Piggott, and Lee Crust. 2012. A systematic review of the experience, occurrence, and controllability of flow states in elite sport. Psychology of sport and exercise 13, 6 (2012), 807-819. DOI :

http://dx.doi.org/10.1016/j.psychsport.2012.05.006

[59] Christian Swann, David Piggott, Lee Crust, Richard Keegan, and Brian Hemmings. 2015. Exploring the interactions underlying flow states: A connecting analysis of flow occurrence in European Tour golfers. Psychology of Sport and Exercise 16 (2015), 60-69. DOI :

http://dx.doi.org/10.1016/j.psychsport.2014.09.007

[60] Krsna Das Thoondee and Clifton Lane. 2017. Using Virtual Reality to Reduce Stress at Work. In Computing Conference. 492-499. DOI :

http://dx.doi.org/10.1109/SAI.2017.8252142

[61] Deltcho Valtchanov, Kevin R Barton, and Colin Ellard. 2010. Restorative effects of virtual nature settings. Cyberpsychology, Behavior, and Social Networking 13, 5 (2010), 503-512. DOI :

http://dx.doi.org/10.1089/cyber.2009.0308 
[62] Jacqueline Vischer. 1999. Will this open space work? Harvard Business Review 77, 3 (1999), 28-40.

[63] Maria Meiha Wong and Mihaly Csikszentmihalyi. 1991. Motivation and academic achievement: The effects of personality traits and the duality of experience. Journal of Personality 59, 3 (1991), 539-574. DOI:

http://dx.doi.org/10.1111/j.1467-6494.1991.tb00259.x

[64] William J Wrigley and Stephen B Emmerson. 2013. The experience of the flow state in live music performance.
Psychology of Music 41, 3 (2013), 292-305. DOI : http://dx.doi.org/10.1177/0305735611425903

[65] Daniel Yaroslavski. 2014. Lightbot. Retrieved on 25th May (2014).

[66] Fred R. H. Zijlstra, Robert A. Roe, Anna B. Leonora, and Irene Krediet. 1999. Temporal factors in mental work: Effects of interrupted activities. Journal of Occupational and Organizational Psychology 72, 2 (jun 1999), 163-185. DOI:

http://dx.doi.org/10.1348/096317999166581 